\title{
Cyclical changes in sperm volume during in vitro incubation under capacitating conditions: a novel boar semen characteristic
}

\author{
A. M. Petrounkina ${ }^{1}$, R. A. P. Harrison ${ }^{2}$, R. Petzoldt ${ }^{3}$, K. F. Weitze ${ }^{1}$ and E. Töpfer-Petersen ${ }^{1 *}$ \\ ${ }^{1}$ Institute for Reproductive Medicine, School of Veterinary Medicine Hannover, Buenteweg 15, 30559 Hannover, Germany; ${ }^{2}$ Laboratory of \\ Gamete Signalling, The Babraham Institute, Babraham, Cambridge CB2 4AT, UK; and ${ }^{3}$ Department of General Ecology, Brandenburg \\ Technical University, Cottbus, Bad Wilsnacker Str. 43, 19322 Wittenberge, Germany
}

\begin{abstract}
The osmotic reactivity of boar spermatozoa during incubation in vitro was studied using a hypo-osmotic swelling test in conjunction with electronic measurement of cell volume. Sperm populations showed fluctuations in both iso-osmotic cell volume and hypo-osmotic volume response that fitted mathematical models for periodicity. Significant differences of frequency and amplitude were observed during sperm incubation under capacitating conditions as compared with those under noncapacitating conditions. In addition, different boars showed specific differences in their fluctuation characteristics under capacitating conditions. During incubation under capacitating conditions, a decrease in osmotic reactivity was observed that correlated with a decrease in motility, while the absolute value of the earliest maximum of the osmotic-induced response correlated with an increase in the proportion of discharged acrosomes. The time course of the cyclical behaviour of osmotic reactivity may be a useful parameter for assessing boar sperm response to capacitating conditions.
\end{abstract}

\section{Introduction}

It is now well established that during capacitation there are major changes in the functional state of the sperm plasma membrane (Yanagimachi, 1994; Töpfer-Petersen et al., 1996). One aspect of these changes is an increasing destabilization which prepares the membrane for the acrosome reaction and fusion with the oocyte (see Harrison, 1996). An important likely consequence of the changes in sperm plasma membrane function is a change in ion transport regulation, resulting in a change in intracellular ionic concentrations. Since spermatozoa appear to behave as perfect osmometers (Drevius, 1972; Du et al., 1994; Gilmore et al., 1996), changes in cell osmole content, as well the distribution of the osmotically active and inactive cells within a sperm population, might be revealed as volume changes of sperm cells under iso-osmotic enmvironmental conditions. However, information about changes in cell osmole content might be more detectable under hypo-osmotic stress, since this would induce a rapid increase of cell volume due to the high water permeability of the spermatozoa (Gilmore et al., 1996, and references therein). Thus, changes of cell volume measured under both iso-osmotic and hypo-osmotic conditions would be an expression of the dynamics of cell water balance as influenced by capacitation treatment. The objective of this study was the establishment and

*Correspondence.

Revised manuscript received 11 October 1999. preliminary testing of an analytical procedure for investigating cell volume dynamics during capacitation. The approach used was based on protocols developed for the application of electronic volume analysis to hypo-osmotic swelling tests (Petzoldt, 1988; Engel and Petzoldt, 1994; Petzoldt and Engel, 1994).

\section{Materials and Methods}

\section{Chemicals}

Unless otherwise stated, all chemicals were obtained from Merck AG (Darmstadt).

\section{Animals and sperm collection}

Semen samples (whole ejaculates) were collected by the gloved-hand method from nine different mature boars (referred to later as boars A-I) of the German Landrace and Schwäbisch-Hällisch breeds housed at the Institute for Reproductive Medicine. Gel material was removed by filtration through sterile gauze and the spermatozoa were subsequently obtained by centrifugation of the sample for $10 \mathrm{~min}$ at $500 \mathrm{~g}$. The pellet was resuspended in residual supernatant seminal plasma to a final concentration of about 1-2 $\times 10^{9}$ cells $\mathrm{ml}^{-1}$. During collection, transfer to the laboratory, and pre-manipulation incubation, the semen was 
maintained at a temperature of at least $25^{\circ} \mathrm{C}$ to avoid cold shock. Samples were used for the experimental studies within $30 \mathrm{~min}$ of collection.

\section{Incubation media}

As a capacitation medium, the complete bicarbonatecontaining Tyrode's-based medium of Harrison et al. (1993) was used (bT); this consisted of $96 \mathrm{mmol} \mathrm{NaCl} \mathrm{l}^{-1}, 3.1 \mathrm{mmol}$ $\mathrm{KCl} \mathrm{l}^{-1}, 2 \mathrm{mmol} \mathrm{CaCl}_{2} \mathrm{l}^{-1}, 0.4 \mathrm{mmol} \mathrm{MgSO} \mathrm{I}^{-1}, 15 \mathrm{mmol}$ $\mathrm{NaHCO}_{3} \mathrm{l}^{-1}, 0.3 \mathrm{mmol} \mathrm{NaH}_{2} \mathrm{PO}_{4} \mathrm{l}^{-1}, 5 \mathrm{mmol}$ glucose $\mathrm{l}^{-1}$, 21.6 mmol sodium lactate $\mathrm{l}^{-1}$ (Sigma-Aldrich Ltd, Steinheim), $1 \mathrm{mmol}$ sodium pyruvate $\mathrm{l}^{-1}, 20 \mathrm{mmol} \mathrm{Hepes} \mathrm{l}^{-1}$ (Serva $\mathrm{GmbH}$, Heidelberg), $3 \mathrm{mg} \mathrm{BSA} \mathrm{m}^{-1}$ (final concentration; fraction V; Sigma product A-8022) and $10 \mu \mathrm{g}$ phenol red $\mathrm{ml}^{-1}$ and the medium was maintained in equilibrium with $5 \%$ $\mathrm{CO}_{2}$. As a control, non-capacitating, medium a Hepesbuffered saline medium (HBS; Harrison et al., 1993) was used; this consisted of $137 \mathrm{mmol} \mathrm{NaCl} \mathrm{l}^{-1}, 10 \mathrm{mmol}$

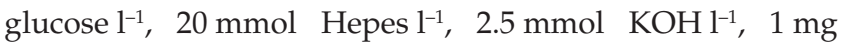
polyvinylalcohol $\mathrm{ml}^{-1}$ (Sigma product P-8136) and $1 \mathrm{mg}$ polyvinylpyrrolidone $\mathrm{ml}^{-1}$ (Sigma product P-7443). Both media had an osmolality of $300 \mathrm{mOsm} \mathrm{kg}^{-1}$, and a $\mathrm{pH}$ value of 7.4. Before use, both media were passed through a $0.2 \mu \mathrm{m}$ filter.

\section{Experimental protocol}

Two investigations were made. Experiment 1 tested the general effect of incubation under capacitating conditions on three separate ejaculates from each of six boars and two separate ejaculates from a further three boars. Experiment 2 tested the specific effect of the capacitating medium on three separate ejaculates from each of three of the same nine boars.

In Expt 1, aliquants of the sperm suspensions were incubated in nine volumes of bT medium for $180 \mathrm{~min}$ under 5\% $\mathrm{CO}_{2}: 95 \%$ air at $39^{\circ} \mathrm{C}$. In Expt 2, sperm samples were incubated in nine volumes of HBS medium for $90 \mathrm{~min}$ at $39^{\circ} \mathrm{C}$, after which the spermatozoa were collected by centrifugation at $500 \mathrm{~g}$ for $10 \mathrm{~min}$ (ambient temperature) and resuspended in a portion of the supernatant. One aliquot of the resulting sperm suspension was diluted in nine volumes of bT medium, another in nine volumes of HBS medium, and each was incubated for a further $90 \mathrm{~min}$ at $39^{\circ} \mathrm{C}$ (the bT suspensions were incubated under $5 \% \mathrm{CO}_{2}$ ).

In both experiments, $4 \mu \mathrm{l}$ samples were taken from the incubation suspension at $15 \mathrm{~min}$ intervals and added to $10 \mathrm{ml}$ of iso-osmotic (300 mOsm kg-1 or hypo-osmotic $\left(150 \mathrm{mOsm} \mathrm{kg}^{-1}\right) \mathrm{NaCl}$ solution contained in snap-cap glass vials (ScharfGlas: Landgraf Laborgeräte, Langenhagen) and preincubated at $39^{\circ} \mathrm{C}$ (Engel and Petzoldt, 1994). The samples were then passed through a CASY 1 cell counter (Schaerfe Systems GmbH, Reutlingen), which produced cell volume information on the basis of cell frequency distribution within 1024 electronic 'volume' channels. At each sampling time-point, such distributions were collected from a single iso-osmotic dilution and a single hypo-osmotic dilution using a CASY sample volume setting of $200 \mu \mathrm{l}$ and a size scale of $10 \mu \mathrm{m}$. Each sampling obtained data from $>8000$ cells.

The volume distributions at all sampling points were measured $30 \mathrm{~s}$ after dilution in the iso-osmotic $\mathrm{NaCl}$ solution, or $3 \mathrm{~min}$ after dilution in the hypo-osmotic solution. These different times of exposure were adopted to allow parallel observations of motility as well as adequate rinsing of the CASY capillary tubing between each volumetric measurement. Thus, the incubation time in the measuring solution remained constant for all sampling points. The first sampling was performed immediately after the initial dilution in incubation medium.

At the start and end of each incubation, estimations were made of the sperm motility (at $39^{\circ} \mathrm{C}$ ) and acrosomal integrity. Sperm morphology was examined in samples taken from the native ejaculates shortly after semen collection. The cells were fixed in formol citrate to assess their morphology and acrosomal state (Hancock 1956) and abnormal forms were classified according to Krause (1966).

\section{Initial treatment of data}

Analysis of the CASY measurements was carried out on the exported numerical data, that is, the count number in each of the 1024 effective particle size channels.

A mathematical approach (see Appendix) was adopted to 'normalize' each data set and allow proper statistical comparisons: (1) Data corresponding to events of small volume (subcellular debris and 'noise') and to events of large volume (probably due to agglutinated sperm) were discarded. This was done by ignoring data in channel numbers below 225 and above 630; these limits were determined from preliminary experiments and remained unaltered for all data sets in the entire study. After this elimination, the resultant data sets still contained $>7000$ events and, in $90 \%$ of cases, $>10000$ events. (2) The area under the resultant distribution curve was calculated by integration, after which the cell number in each channel involved in the distribution curve was divided by this area. This procedure (approximately equivalent to dividing the cell number in each channel by the total number of cells in the selected data) enabled the volume distribution to be considered as a probability function of fixed value that was independent of the number of sperm cells involved in the actual measurement. This was an important consideration for valid application of the subsequent statistical tests of differences between curve shapes.

Because the two volume test solutions had different electrical conductivities (different concentrations of electrolyte), it was necessary to apply a correction factor (1.19 $\pm 0.07)$ to data recorded under hypo-osmotic conditions. This factor was obtained by comparison of volume measurements of standard latex beads ( $3.4 \mu \mathrm{m}$ in diameter; Sigma) in the two solutions. A calibration factor for the CASY could be obtained from the same measurements, which related the modal peak value of the bead volume measurements on the nominal $10 \mu \mathrm{m}$ scale of the machine to the given diameter of the beads; this factor $(F)$ was used to calculate the sperm volume equivalents in physiological terms (see Appendix equations $\mathrm{A} 6$ and $\mathrm{A7}$ ). 


\section{Analysis of the volume distribution curves}

Ejaculate variation within boars. The Kolmogorov-Smirnoff two-sample test (Young, 1977; Müller, 1991) was used to compare volume distributions obtained from replicate ejaculates during incubation under capacitating conditions; pair-wise comparisons were made within sampling timepoints (that is, both hypo-osmotic and iso-osmotic distributions from ejaculate 1 from boar $\mathrm{A}$ at sampling point 1 were compared with parallel distributions from ejaculate 2 from boar A at sampling point 1; ejaculate 1 was compared with ejaculate 3 , and ejaculate 2 with ejaculate 3 , also at sampling point 1 ; the whole procedure was then repeated for sampling point 2, and so on). Three ejaculates were considered from each of five boars (A-E).

Building of average volume distributions. Two sets of 'average volume distributions' were obtained for each incubation system (one for iso-osmotic response and one for hypoosmotic response) to investigate the effects of incubation under capacitating and non-capacitating conditions. These average volume distributions were constructed by averaging for each 'volume' channel the normalized frequencies from the trials of three different ejaculates from the same boar. Thus, average distributions were obtained for each of the sampling time points involved in the two incubation systems.

Boar response to capacitating conditions. Differences among boars with respect to the shapes of their average volume distributions during incubation under capacitating conditions were examined by means of the KolmogorovSmirnoff test. Five boars were compared in a pairwise fashion; at each sampling point, the average hypo-osmotic distribution for boar A was compared with that for boar B, likewise the average iso-osmotic distribution, and so on.

\section{Analysis of derived volumetric parameters}

Definition of volumetric parameters. The volumetric parameters used are described (Fig. 1).

The relative volume shift $V_{\mathrm{r}}$ was used as a measure of the effect of hypo-osmotic stress and was defined on the basis of the iso-osmotic and hypo-osmotic volume distributions (Petzoldt and Engel, 1994):

$$
V_{\mathrm{r}}=V_{\text {hypo }} / V_{\text {iso }}
$$

where $V_{\text {hypo }}$ is the modal value of the hypo-osmotic volume distribution, and $V_{\text {iso }}$ is the modal value of the iso-osmotic volume distribution. In a situation in which several sperm subpopulations contributed to a distribution, the values pertaining to the dominant osmotically active subpopulation were used. A cell subpopulation was considered as osmotically active if its $V_{\mathrm{r}}>1$.

Time series analysis. Because the osmotic volume responses $V_{\mathrm{r}}$ and $V_{\text {iso }}$ appeared to show periodic fluctuations, their changes with time were investigated using time series analysis, a model of periodic fluctuations (see Appendix). Application of this procedure provided amplitude and
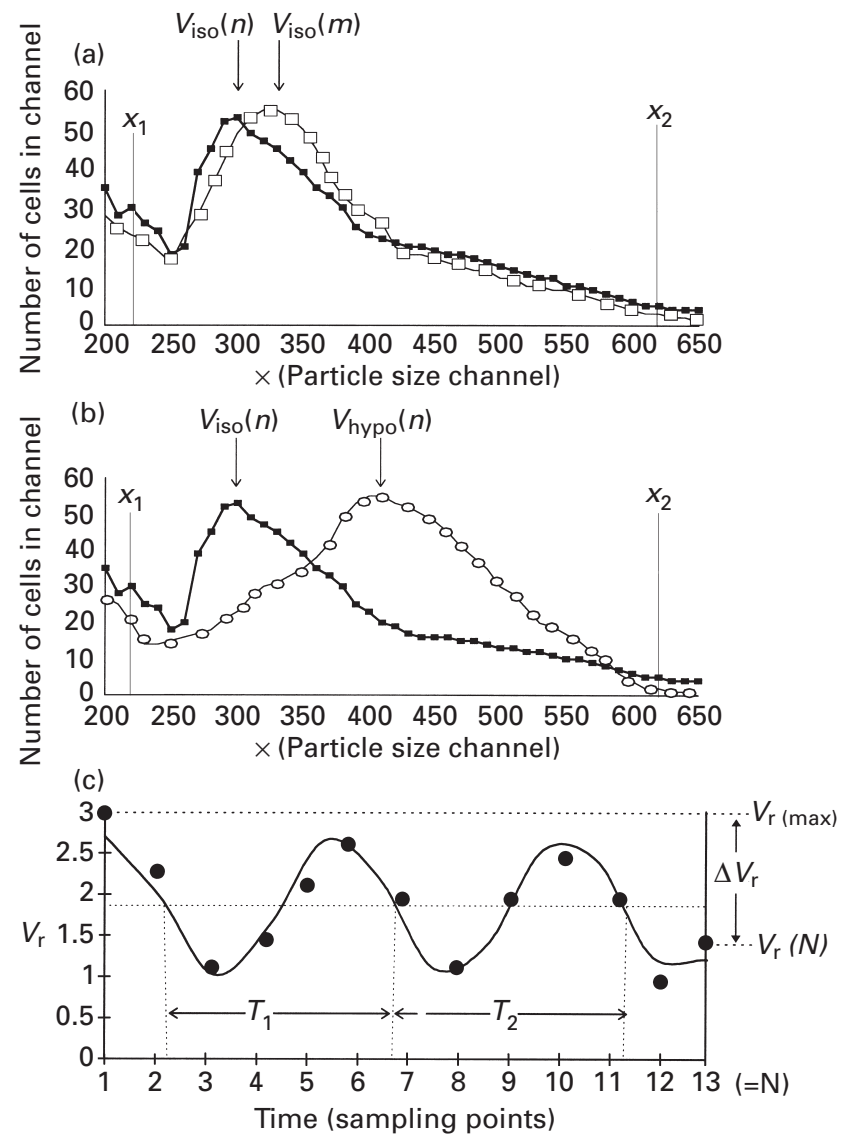

Fig. 1. Diagrammatic definition of volumetric parameters of boar sperm populations. (a) Effect of incubation (from sampling time point $n$ to sampling time point $m$ ) on iso-osmotic volume distribution. $V_{\text {iso }}=$ modal value of the iso-osmotic volume distribution. $\square$ : Distribution at time-point $n ; \square$ : distribution at timepoint $m$. (b) Effect of hypo-osmotic stress at sampling time-point $n$. $V_{\text {hypo }}=$ modal value of the hypo-osmotic volume distribution. $\mathbf{\square}$ : Isoosmotic volume distribution; $\bigcirc$ : hypo-osmotic volume distribution. (c) Time-based cyclical fluctuations in osmotic response. $V_{\mathrm{r}^{\prime}}$ the relative volume shift $=V_{\text {hypo }} / V_{\text {iso }} ; V_{\mathrm{r}}(n)=V_{\mathrm{r}}(0), V_{\mathrm{r}}(1), \ldots, V_{\mathrm{r}}^{\mathrm{r}^{\prime}}(\mathrm{N})$, that is, the relative volume shift changes at the different sampling time points, where $n=1 \ldots \mathrm{N}, 1$ being the first sampling point (at $t=0 \mathrm{~min}$ ) and $\mathrm{N}=$ the last sampling point (at the end of the incubation period). The points are the actual individual $V_{\mathrm{r}}$ values for the particular incubation series ('averaged' data from ejaculate replicates for a given boar). The curve represents the derived time analysis model from the sequence of $V_{\mathrm{r}}$ values (see Appendix, equations (A3), (A4) and (A5)); $T_{i}=i$-th cycle duration of $V_{\mathrm{r}}$ (or $V_{\text {iso }}$ ) fluctuations $\left(T_{1}\right.$ is the first, etc.) $V_{\mathrm{r}}(\max )=$ the earliest maximum of $V_{\mathrm{r}}$ (actual value for a given boar); $\Delta V_{\mathrm{r}}=V_{\mathrm{r}}(\max )-V_{\mathrm{r}}(\mathrm{N})$.

frequency values, the main characteristic parameters of rhythmic biological fluctuations.

Normalization of the model functions. The oscillations of $V_{\mathrm{r}}$ and $V_{\text {iso }}$ fitted to the model of periodical fluctuations were normalized using a similar procedure to that described for the volume distribution data, so that the time-based functions of osmotic response could be considered as a probability function of fixed value. In this way, generalized 
comparisons of the time response could be carried out using a Kolmogorov-Smirnoff test as described above.

\section{Other analytical procedures}

The influence of animals and incubation time on conventional sperm parameters and on derived volumetric parameters during incubation under capacitating conditions were examined by analysis of variance using a general linear procedure of the SAS-Program (cross-classification with repetitions, Müller, 1991; SAS, 1989). Relationships among different spermatological parameters (including the volumetric parameters) were tested by Pearson correlation analysis.

\section{Miscellaneous}

Calculations were performed by the use of CASY, Excel, and SAS software; SAS-language-based programmes were written to enable data standardization and time series analysis.

\section{Results}

\section{Conventional sperm parameters}

Descriptive statistics of the conventional sperm parameters for native ejaculates and for sperm samples at the start and end of incubation in capacitation medium for the nine boars involved in the study are listed (Table 1). The motility characteristics of samples during incubation in either capacitating or control medium after preincubation in a non-capacitating medium are also shown (Table 2). The values indicate that, in general and despite differences between the various samples, the ejaculates from the nine boars were of good quality as judged by conventional parameters.

\section{Individual boar differences in volume response}

Within-boar comparisons of volumetric distribution curves were made on groups of three ejaculates from each of five boars (A-E) using the Kolmogorov-Smirnoff two-sample test to test whether valid 'average' data could be constructed for the individual boars. No significant differences in curve shape were seen between the three ejaculates in such groups in 56 of 65 trials of iso-osmotic distribution tests and in 55 of 65 trials of hypo-osmotic distribution tests (within-triplet trials of data for each of 13 sampling time points for each of five boars =65).

When inter-boar comparisons were made on the same five boars (using average values for their three ejaculates), two of the five boars ( $\mathrm{B}$ and $\mathrm{D})$ showed significant differences $(P<0.05)$ from the others in their iso-osmotic cell volume distributions at the start of incubation in the capacitation medium. Three of the five boars $(\mathrm{B}, \mathrm{D}$ and $\mathrm{E})$ showed significant differences $(P<0.05)$ from each other and from the other two in their hypo-osmotic response at the start of incubation. These same boars also showed significant differences in either iso-osmotic or hypoosmotic responses during such incubation $(P<0.05)$. Two types of individual cell volume distribution response to hypo-osmotic conditions in boars having similar types of iso-osmotic volume distribution curve (boars $\mathrm{A}$ and $\mathrm{E}$ ) are shown (Fig. 2). The shapes of the hypo-osmotic volume distributions were significantly different $(P<0.05)$.

These analyses validated the construction of 'average' volumetric data sets and indicated that the volumetric tests used in the present study were sufficiently sensitive to reveal differences among boars.

Table 1. Descriptive statistics of conventional parameters of the boar sperm samples used in Expt 1

\begin{tabular}{|c|c|c|c|}
\hline & $\operatorname{Mean}^{*} \pm \mathrm{SD}(n=9)$ & Range $^{a}$ & Number of ejaculates \\
\hline Percentage of spermatozoa motile in native semen & $89.2 \pm 5.7$ & $78-95$ & 24 \\
\hline Percentage of spermatozoa motile at start of incubation ${ }^{b}$ & $83.9 \pm 5.9$ & $73-90$ & 24 \\
\hline Percentage of spermatozoa motile at end of incubation ${ }^{\mathrm{b}}$ & $59.6 \pm 22.4$ & $15-80$ & 24 \\
\hline Percentage of normal forms in native semen & $84.6 \pm 11.3$ & $59-96$ & 24 \\
\hline Decrease in percentage of normal acrosomes during incubation & $14.9 \pm 2.0$ & $12-18$ & $19^{c}$ \\
\hline
\end{tabular}

*Overall mean \pm SD for all nine boars

avalues are means for individual boars.

'Incubation under capacitating conditions.

'Five missing values.

Table 2. Descriptive statistics of the boar sperm motility changes observed during Expt 2 (incubation in control and capacitating medium after preincubation in control medium)

\begin{tabular}{lrr}
\hline & Control medium & Capacitating medium \\
\hline Percentage of spermatozoa motile at start of second incubation & $82.7 \pm 2.5$ & $82.9 \pm 2.6$ \\
Percentage of spermatozoa motile at end of second incubation & $78.9 \pm 1.0$ & $70.0 \pm 1.6^{*}$ \\
\hline
\end{tabular}

Values are mean $\pm \mathrm{SD}$ for nine ejaculates.

*Significantly lower than in control medium $(P<0.05)$. 


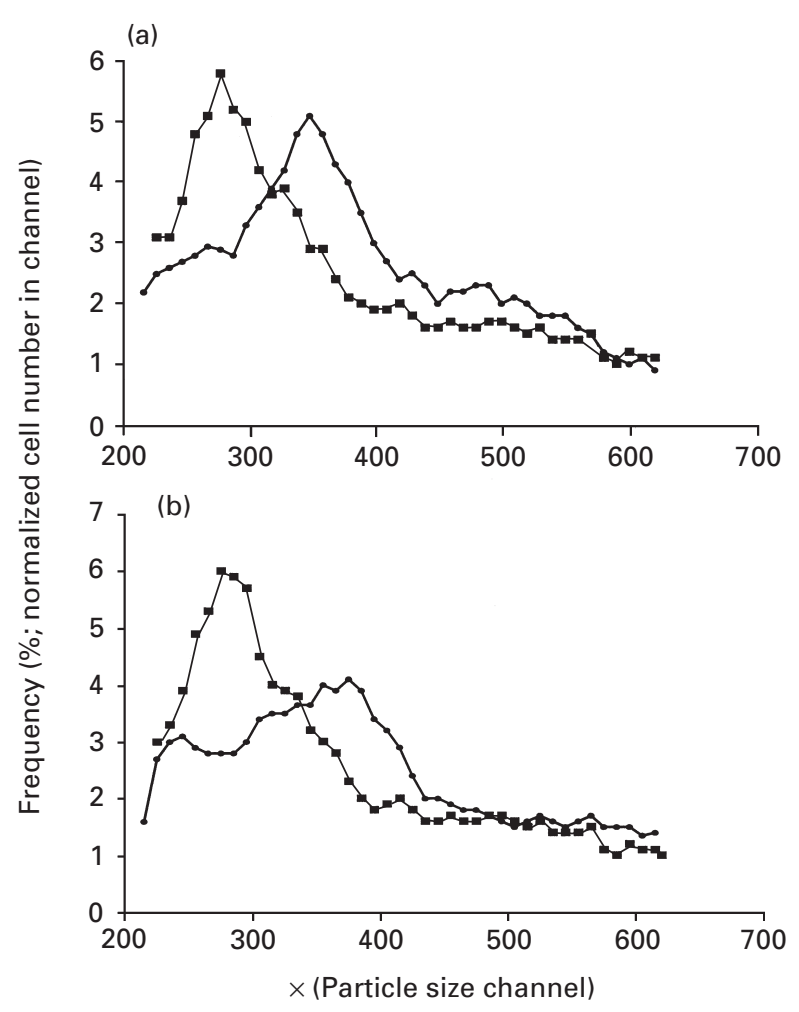

Fig. 2. Two types of boar sperm cell volume distribution response to hypo-osmotic stress. 'Average' distributions (obtained at start of incubation) are presented for two boars that showed similar responses to iso-osmotic dilution. - - - : iso-osmotic dilution; -0-: hypoosmotic dilution. (a) Boar A: after hypo-osmotic dilution, the spermatozoa showed a discrete population distribution with increased volume. (b) Boar E: after hypo-osmotic dilution, the spermatozoa showed a more broadly dispersed population with increased volume. The Kolmogorov-Smirnoff test showed no significant difference between the iso-osmotic volume distributions of these two boars, whereas the hypo-osmotic volume distributions were significantly different $(P<0.05)$. $V_{\mathrm{r}}$ was 2.11 in (a) and 3.14 in (b).

\section{Temporal dynamics of iso-osmotic volume and hypo-osmotic response}

Inspection of average volumetric data obtained for nine boars revealed that the modal values $V_{\text {iso }}$ and $V_{\text {hypo }}$ fluctuated during incubation. An example of the temporal dynamics of $V_{\text {iso }}$ during incubation in capacitation medium is shown (Fig. 3). Therefore, time series analysis was applied to $V_{\text {iso }}$ and to the derived osmotic response parameter $V r$, to test whether their changes could be fitted to a periodic fluctuation model (see Appendix). Comparing the experimental data with the calculated model trigonometric functions (see Appendix) by stepwise multiple regression analysis, the fit was found to be significant $(P<0.05$ for the null hypothesis that there was no evidence of fit) for all nine boars, both for $V_{\text {iso }}$ and for $V_{\mathrm{r}}$. Therefore, the time course of cellular osmotic reactivity appeared to represent a multimodal but cyclic event and could be approximated by a linear combination of trigonometric functions.

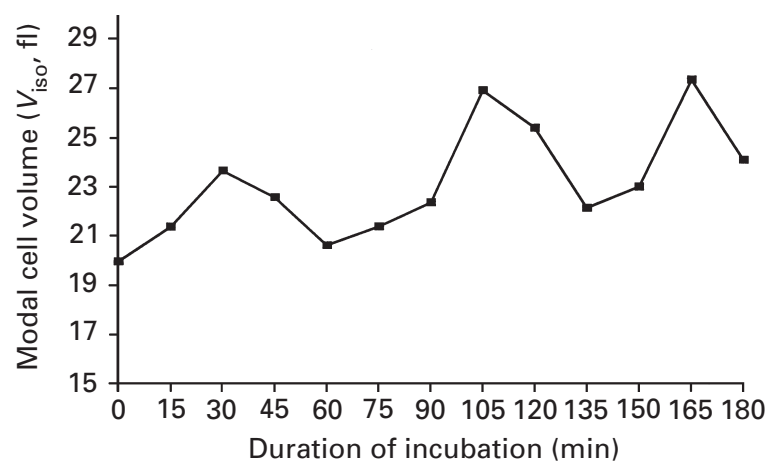

Fig. 3. Typical example of cyclical variation in iso-osmotic volume during incubation of boar spermatozoa under capacitating conditions. The data shown are for boar C (obtained from 'average' distributions). $V_{\text {iso' }}$ the modal value of the iso-osmotic volume distribution, has been expressed in femtolitre (fl) equivalents (calculated by comparison with measurements on standard latex beads), and the time-base has been expressed as minutes of incubation to relate the data to more 'physiological' parameters.

\section{Grouping of boars with respect to characteristics of cyclical volumetric response}

The numerical values for the volumetric parameters obtained during incubation in capacitating medium were compared among individual boars. Applying equations A4 and A5 of the Appendix, two significantly different $(P<0.05)$ $V_{\mathrm{r}}$ time-course shapes were seen: six of the nine boars $(\mathrm{A}, \mathrm{B}$, E, F, G, and H) showed one type, the other three boars (C, D and I) showed another type. The volumetric parameters and associated conventional spermatological parameters for the two groups of boars are listed (Table 3). The groups showed no difference in either mean $V_{\text {iso }}$ itself or the periodicity of $V_{\text {iso }}$ cyclicity. (The calculation of $V_{\text {iso }}$ as a volume equivalent (Table 3; Fig. 3) yielded values in close agreement with other estimates of boar sperm volume, for example those of $\mathrm{Du}$ et al. (1994)). However, there were significant differences in $V_{\mathrm{r}} \max$ and in the cyclical frequency of $V_{\mathrm{r}}$ changes, as well as a significant difference with respect to decrease of motility during the incubation period. The correlation matrix of volumetric versus conventional sperm parameters is shown (Table 4). $V_{\mathrm{r}} \max$ correlated significantly with the duration of the first $V_{\mathrm{r}}$ cycle and also with acrosomal deterioration, while the percentage decrease of $V_{\mathrm{r}}$ correlated both with the duration of the first cycle and with the decrease in motility. The duration of the first cycle correlated both with decrease in motility and with acrosomal deterioration.

\section{Media effects}

The comparative effects of capacitating (bT) and noncapacitating (HBS) medium on volumetric parameters of three boars are shown (Table 5). Two types of comparison were made for the two media. In the first, the behaviour of spermatozoa during the first $90 \mathrm{~min}$ of incubation in bT during Expt 1 was compared with behaviour of spermatozoa (from the same animals) during the initial incubation in HBS during Expt 2. All three boars involved showed significant 
Table 3. Comparison of derived volumetric and conventional sperm parameters for two groups of boars with respect to the response to capacitating conditions

\begin{tabular}{|c|c|c|}
\hline & Group $1(n=6)^{\mathrm{a}}$ & Group $2(n=3)^{\mathrm{b}}$ \\
\hline$V_{\mathrm{r}} \max$ & $2.8 \pm 0.2$ & $3.2 \pm 0.3^{*}$ \\
\hline Relative decrease in $V_{\mathrm{r}}$ during incubation $\left(100 \times \Delta V_{\mathrm{r}} / V_{\mathrm{r}} \max \right)$ & $10.6 \pm 6.3$ & $21.9 \pm 4.6^{*}$ \\
\hline \multicolumn{3}{|l|}{ Interval lengths ( $\mathrm{min}$ ) of $V_{\mathrm{r}}$ cycles (see Fig. 1c) } \\
\hline First cycle $\left(T_{1}\right)$ & $41 \pm 12$ & $62 \pm 3^{*}$ \\
\hline Second cycle $\left(T_{2}\right)$ & $36.5 \pm 9$ & $44 \pm 9$ \\
\hline Third cycle $\left(T_{3}\right)^{c}$ & $38.5 \pm 11$ & $32 \pm 5$ \\
\hline$V_{\text {iso }}$ (femtolitre equivalents) ${ }^{\mathrm{d}}$ & $22.2 \pm 2.5$ & $21.3 \pm 2.1$ \\
\hline Mean interval length $(\mathrm{min})$ for $V_{\text {iso }}$ cycles $^{\mathrm{e}}$ & $47 \pm 9$ & $46 \pm 7$ \\
\hline \multicolumn{3}{|l|}{ Motility (\%) } \\
\hline in native semen & $90.3 \pm 4.8$ & $87.2 \pm 7.9$ \\
\hline at start of incubation $\left(\mathrm{M}_{0}\right)$ & $84.2 \pm 4.9$ & $83.3 \pm 8.8$ \\
\hline at end of incubation $\left(\mathrm{M}_{\mathrm{N}}\right)$ & $72.0 \pm 7.4$ & $53.8 \pm 22.0$ \\
\hline Relative decrease in motility during incubation $\left(100 \times\left[\mathrm{M}_{0}-\mathrm{M}_{N}\right] / \mathrm{M}_{0}\right)$ & $15.3 \pm 7.0$ & $36.8 \pm 20.5^{*}$ \\
\hline \multicolumn{3}{|l|}{ Morphology } \\
\hline Percentage of normal forms in original ejaculate & $85.1 \pm 13.4$ & $83.5 \pm 7.6$ \\
\hline Decrease in percentage of normal acrosomes during incubation & $14.2 \pm 1.9$ & $16.6 \pm 1.6$ \\
\hline
\end{tabular}

Values are means \pm SD.

$V_{\mathrm{r}^{\prime}}$ relative volume shift; $V_{\text {iso }}$, modal value of the iso-osmotic volume distribution.

${ }^{\mathrm{a}}$ Group 1: boars A, B, E, F, G and H; ${ }^{\mathrm{b}}$ Group 2: boars C, D and I

'For boars $D$ and $E$, values of double the half-cycle duration, that is $2 \times\left[T_{3}(1 / 2)\right]$, were used because the full cycle was not completed by the end of incubation.

${ }^{\mathrm{d}}$ For each boar, the $V_{\text {iso }}$ value was for the same time-point at which its $V_{\mathrm{r}}$ max was detected.

eAnalysis of $V_{\text {iso }}$ fluctuations showed that variations between successive cycle durations were much less than fluctuations for $V_{\mathrm{r}}$

These values were obtained by linear regression calculation using pooled data (see Appendix, paragraph after equation A5).

${ }^{*}$ Group 2 significantly different from Group $1(P \leqslant 0.05)$.

Table 4. Correlation matrix for conventional and derived volumetric parameters for boar sperm cell suspensions incubated under capacitating conditions (Expt 1)

\begin{tabular}{llcccc}
\hline & $\mathrm{d} V_{\mathrm{r}}$ & $T_{1}$ & $\mathrm{M}_{0}$ & $\mathrm{dM}$ & $\mathrm{dAcr}^{\mathrm{a}}$ \\
\hline$V_{\mathrm{r}}$ max & 0.53 & $0.78^{*}$ & -0.09 & 0.51 & $0.97^{* *}$ \\
Percentage decrease in $V_{\mathrm{r}}\left(\mathrm{d} V_{\mathrm{r}}\right)$ & 1.0 & $0.67^{*}$ & -0.35 & $0.87^{* *}$ & 0.70 \\
Duration of first $V_{\mathrm{r}}$ cycle $\left(\mathrm{T}_{1}\right)$ & & 1.0 & -0.3 & $0.75^{*}$ & $0.86^{*}$ \\
Motility at start $\left(\mathrm{M}_{0}\right)$ & & & 1.0 & -0.55 & -0.46 \\
Percentage decrease in motility $(\mathrm{dM})$ & & & & 1.0 & $0.88^{*}$ \\
\hline
\end{tabular}

$V_{\mathrm{r}^{\prime}}$ relative volume shift

a Decrease in percentage of normal acrosomes

${ }^{*} P \leqslant 0.05,{ }^{*} P \leqslant 0.001$.

differences between the two media with respect to periods of oscillation of $V_{\mathrm{r}}$ (section (a) in Table 5). Moreover, periods of oscillation for $V_{\text {iso }}$ could be calculated by applying the equations $t_{m}=-1.74+1.56 m$ for incubation in $\mathrm{bT}$ and $t_{m}=-1.35+1.85 m$ for incubation in HBS (where $m$ is the order number of a $V_{\text {iso }}$ maximum or minimum, and $t_{\mathrm{m}}$ is the time point of appearance of this particular extreme; details relating to the derivation of these equations are given in the Appendix). This calculation had to be performed using pooled data from the three boars and could be made only for $V_{\text {iso }}$ fluctuations, for which variation in cycle duration between successive cycles and inter-boar differences between the three particular boars were not significant. It yielded $T=47 \mathrm{~min}$ in capacitating medium and $T=56 \mathrm{~min}$ in control medium (significantly different, $P<0.05$ ).

The second type of comparison considered behaviour in Expt 2, in which direct comparisons could be made on split samples from the same ejaculate during $90 \mathrm{~min}$ incubation either in bT medium or in HBS medium, after preincubation in HBS. All three boars showed a significant overall decline in $V_{\mathrm{r}}$ during incubation in capacitating medium after $90 \mathrm{~min}$ preincubation in control medium, as compared with their behaviour during incubation in the control medium (see Table 5, section (b), where the mean ratio of $V_{\mathrm{r}}$ in HBS to $V_{\mathrm{r}}$ in $\mathrm{bT}$ is given). A lower (or decreasing) $V_{\mathrm{r}}$ in $\mathrm{bT}$ is detected as a significant deviation of the ratio from 1 . Although the 
Table 5. Comparison between capacitating (bT) and non-capacitating (HBS) media with respect to volumetric parameters of boar spermatozoa

\begin{tabular}{|c|c|c|c|}
\hline & Boar A & Boar B & Boar C \\
\hline \multicolumn{4}{|c|}{ (a) Frequency solutions to periodic model equation $\mathrm{A}^{\mathrm{a}}$} \\
\hline HBS & $\{3,4,9\}$ & $\{3,5,6,7\}$ & $\{3,6,7,8\}$ \\
\hline $\mathrm{bT}$ & $\{3,4,5,6,7,8\}^{*}$ & $\{4,6,7,9\}^{*}$ & $\{3,4,5,6\}^{*}$ \\
\hline (b) $V_{\mathrm{r}}$ in HBS $/ V_{\mathrm{r}}$ in $\mathrm{bT}^{\mathrm{b}}$ & $1.22 \pm 0.14^{* *}$ & $1.44 \pm 0.42^{* *}$ & $1.31 \pm 0.52$ \\
\hline
\end{tabular}

a Comparison of the initial 90 min incubation in Hepes-buffered saline medium (HBS) during Expt 2 with first 90 min incubation in bicarbonate-containing Tyrode's-based medium (bT) during Expt 1. From each incubation, a time series of experimental values of $V_{\mathrm{r}}$ (relative volume shift) was obtained for which a set of solutions of equations A3 and thence A4 in the Appendix was obtained; each frequency $\lambda_{k}$ fits the equation $\lambda_{k}=2 \pi n \lambda$, where $n$ is a natural number $(2,3,4$ etc.) and $\lambda$ is the base frequency. The set of numbers given are the values of $\mathrm{n}$ satisfying the fit of $V_{\mathrm{r}}$ cyclic frequency to the periodic model.

birect comparison of $V_{\mathrm{r}}$ in HBS with $V_{\mathrm{r}}$ in bT for each sampling time point during the $90 \mathrm{~min}$ of second incubation in Expt 2 (that is, on split samples) (Means $\pm \mathrm{SD} ; n=7)$.

*Significantly different from HBS ( $P<0.05$ by Kolgomorov-Smirnoff comparison of the time series of $V_{r}$ changes in the two media). ${ }^{*}$ Significantly different $(P<0.05)$ from 1 (that is, from the null hypothesis that there was no difference between $\mathrm{bT}$ and HBS).

deviation overall was not significant for boar $C$, the deviation became significant at later sampling time points. The decrease in motility during incubation in bT was also significantly greater than it was in HBS (see Table 2).

\section{Response of boar E to capacitating conditions}

Although only minor differences were seen among semen samples in terms of conventional sperm parameters, and none were of poor initial quality, one of the boars (E) showed especially rapid changes in volumetric response to capacitating conditions. In boar E, two modes (that is, two subpopulations) could be distinguished within the hypoosmotic volume distributions after $75 \mathrm{~min}$ of incubation for all three ejaculates tested, one with $V_{\mathrm{r}}>1$, the other with $V_{\mathrm{r}}<1$; in one of the ejaculates, two peaks became visible immediately after the start of incubation. Only the values of the osmotically active cell fraction $\left(V_{\mathrm{r}}>1\right)$ were used for temporal modelling. The $V_{\mathrm{r}}$ time course for both subpopulations is shown (Fig. 4) and, whereas the osmotically inactive subpopulation showed no volume fluctuations, the osmotically active subpopulation continued to exhibit regular cyclicity throughout, although its numbers decreased in proportion to the whole from about 90 to about $65 \%$ during the incubation.

\section{Discussion}

This study used a CASY cell counter to collect electronic volume data for several thousand individual cells within samples from boar sperm suspensions to monitor changes in cell volume during incubation under capacitating and noncapacitating conditions. All other things being equal, cell volume essentially reflects the water balance between the inside and outside of the cell, which in turn reflects the ionic distribution inside and outside the cell (for review, see Hoffmann and Dunham, 1995). Such distribution depends not only on the controlled passage of ions through plasma membrane ion channels, but also on the activity of energydependent ion pumps working against natural ion

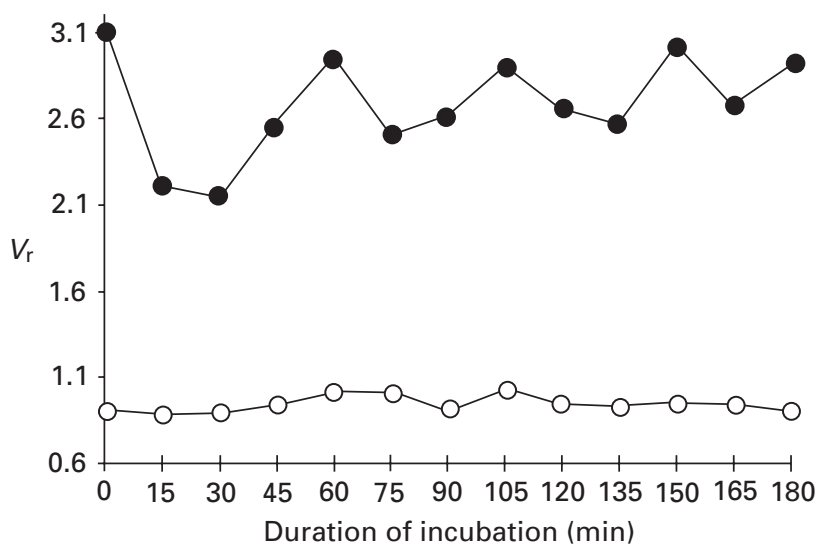

Fig. 4. Osmotic response of two visible boar sperm subpopulations within the same cell suspension incubated under capacitating conditions. The data relate to boar $\mathrm{E}$, in which the ejaculates exhibited two modal values in their hypo-osmotic volume distributions. - - $V_{\mathrm{r}^{\prime}}$ the relative volume shift, of the osmotically active cell subpopulation; - $\bigcirc-$ : $V_{\mathrm{r}}$ of the osmotically inactive cell subpopulation.

concentration differences between the cell interior and the surrounding environment. Many studies have provided evidence that during capacitation, which prepares the sperm for interaction with the egg, major changes in plasma membrane function occur (for reviews, see Yanagimachi, 1994; Harrison, 1996; Töpfer-Petersen et al., 1996). Because sperm cells are 'perfect osmometers' (Drevius, 1972; Du et al., 1994; Gilmore et al., 1996), the present study was undertaken to test the hypothesis that such changes in plasma membrane function are monitored through the resulting cell volume changes. It was further hypothesized that changes in plasma membrane characteristics are also monitored more sensitively via cell volume response to hypo-osmotic stress. Therefore, two tests were applied: measurement of cell volume under iso-osmotic conditions and measurement of cell volume after controlled exposure to hypo-osmotic conditions.

The sperm samples provided a broad distribution of 
volume 'events' across the 1024 electronic channels of the CASY cell counter. Events at the lower extreme were undoubtedly due to non-cellular particulate matter, while events at the higher extreme were most likely due to cellular aggregates; therefore, these extremes were excluded from further analysis. A spread of individual cell volumes was detected in between these extremes. Although this spread may have been due in part to the highly asymmetric shape of the spermatozoa, it was clear that it reflected other, more specific, characteristics. Using the Kolmogorov-Smirnoff test, distributions could be compared sensitively: replicate ejaculates within boars showed consistent distributions whereas there were significant differences among boars. Therefore, 'average' distributions for the individual boars could be constructed, which made it possible to follow the volume changes of their spermatozoa during incubation.

Despite the spread of detectable volumes for individual cells within sperm samples, the distributions always exhibited a mode that reflected the 'most likely' cell volume value within the sample. Therefore, further analysis was based on these modal values. The modal volume under isoosmotic conditions $\left(V_{\text {iso }}\right)$ was used to track cell volume behaviour itself, while a derived function $\left(V_{\mathrm{r}^{\prime}}\right.$ the relative increase in modal volume following hypo-osmotic stress) was used to track overall changes in plasma membrane characteristics. At the start of investigations, sperm cell volume changes of a gradual and progressive nature were expected as the sperm plasma membrane underwent the slow overall change of capacitation. However, while there was indeed an overall gradual change in volume characteristics, both parameters revealed unexpectedly cyclical changes. The periodicity and amplitude of these cyclical changes was enhanced by capacitating conditions. Moreover, there was significant inter-boar variation.

Attempts were made to validate these unexpected findings further. Sperm volume distributions might have been affected by the use of $\mathrm{K}^{+}$-free $\mathrm{NaCl}$ solutions as the osmotic testing media, since Bredderman and Foote (1971a) concluded that the presence of external $\mathrm{K}^{+}$was necessary for volume stabilization in bull spermatozoa. However, boar sperm volume distributions obtained in $\mathrm{NaCl}$ solutions were found to be statistically indistinguishable (by the Kolmogorov-Smirnoff test) from those obtained in HBS (which contained $2.5 \mathrm{mmol} \mathrm{K}^{+} \mathrm{l}^{-1}$, close to the optimum value reported by Bredderman and Foote, 1971a), regardless of whether the solutions were iso- or hypo-osmotic (this finding was valid only for the short exposure times of the volumetric test protocols used).

Spermatozoa adhere to surfaces (Harrison et al., 1978, 1982). Therefore, it seemed possible that, in the present study, cell samples were being measured that were enriched in a moribund sperm subpopulation that did not adhere to the glass surface of the diluting chambers. However, volume distribution curves in hypo-osmotic $\mathrm{NaCl}$ were indistinguishable from distributions in HBS, which contains polyvinyl alcohol and polyvinylpyrollidone as a means of preventing adhesion to surfaces (see Harrison, 1988). Moreover, the numbers of cells contributing to these curves did not differ significantly between $\mathrm{NaCl}$ solutions and $\mathrm{HBS}$, whether samples were from spermatozoa suspended in HBS or in bT, which contains BSA, itself a preventative of adhesion to surfaces (Harrison et al., 1982).

An alternative source of artefactual bias might have been the tendency of live boar spermatozoa to agglutinate, especially in capacitating media (see Harrison, 1997). If increasing numbers of a specific subpopulation of spermatozoa had tended to agglutinate as a consequence of capacitation, the discarding of large events from the analyses would have left data pertaining to an increasingly unrepresentative sperm population. Although there were inevitable variations in the number of cellular events that provided the volume distribution curves for analysis, these variations neither fitted the periodic models derived for the volume changes nor showed as great variation as did the volume changes. Therefore, no evidence was found to suggest that the observations reported here were due to artefacts of experimentation or analysis.

Although there have been numerous estimations of sperm volume and studies of sperm response to osmotic shock, to our knowledge only one other study of time-related sperm volume changes during incubation in vitro has been carried out (Bredderman and Foote, 1971a,b). In this work, evidence was provided that increases in bull sperm volume were associated with cell deterioration; sperm volume and longterm survival was maintained through energy-dependent mechanisms, apparently via the activity of the plasma membrane $\mathrm{Na}-\mathrm{K}$ exchanger. However, Bredderman and Foote (1971a,b) sampled at widely spaced intervals during incubation, and noted no cyclical changes.

Changes in $V_{\text {iso }}$ could be taken as indicating changes in osmotic conditions within the cell, that is, changes in intracellular ionic composition. Ionic conditions within the cell are maintained via a balance between flow (usually inward) through ion channels (specific or non-specific) and the energy-dependent activity of ion transport systems (usually outward). An exception to the inward-outward concept is $\mathrm{K}^{+}$, which tends to exit the cell via $\mathrm{K}^{+}$channels and is recovered through inward transport by the $\mathrm{Na}-\mathrm{K}$ exchanger. The Na-K balance in cells is particularly important since it regulates the steady-state plasma membrane potential, which in turn can affect the degree to which voltage-dependent ion channels are open or closed. A range of ion channels and exchange mechanisms have been identified in spermatozoa, hence changes in the activity or efficacy of these could affect the osmotic conditions within the cell.

The volume of the cell, as well as reflecting intracellular ionic conditions and membrane ion transport function, also reflects the resistance of the plasma membrane to stretching or deformation. This resistance may be due to membrane potential, to the state (or structural arrangement) of the lipid within the plasma membrane bilayer, or to interaction between the plasma membrane and the underlying actin cytoskeleton ('membrane skeleton'; see Fox and Boyles, 1988). In spermatozoa, the resting membrane potential is relatively small and is not strongly $\mathrm{K}^{+}$-dependent (see Rink, 1977); however, Zeng et al. (1995) showed that the membrane potential in mouse spermatozoa becomes more negative during capacitation, as a result of which activation of voltage-sensitive ion channels may become more likely (see 
Florman et al., 1999). Studies by Harrison et al. (1996) have shown that, in boar spermatoa, the structural arrangement of lipid also changes when the spermatozoa are exposed to capacitating conditions. Knowledge of cytoskeletal activity in spermatozoa is less clear. A range of cytoskeletal elements has been detected in spermatozoa, but membrane skeletal structures have not been identified; indeed, there is much variation with respect to reports of actin distribution within spermatozoa (see Moreno-Fierros et al., 1992). Direct evidence of interaction between an actin cytoskeleton and the plasma membrane is sparse, but measurements by Spungin et al. (1995) showed an increase in membraneassociated filamentous actin during capacitation. CastellaniCeresa et al. (1992) have presented evidence that filamentous actin is associated with sub-membranous structures in boar spermatozoa, and that induction of the acrosome reaction with a $\mathrm{Ca}^{2+}$-ionophore induces actin polymerization and attachment of the resultant filamentous actin to detergentinsoluble sperm structures. Moreno-Fiennes et al. (1992) presented data from guinea-pig spermatozoa indicating that rates of polymerization and depolymerization of actin varied considerably in different cellular regions. Therefore, cyclical changes in $V_{\text {iso }}$ observed in the present study may reflect changes in the membrane skeleton resulting from changes in the state of actin, which either affect membrane mechanical resistance directly or affect ion transport mechanisms (see Mills and Mandel, 1994).

The large increase in modal volume caused by exposure to hypo-osmotic stress (expressed by the derived function $V_{\mathrm{r}}$ ) was a sensitive sperm parameter, showing large cyclical changes during incubation and large differences among boars. Although changes in $V_{\mathrm{r}}$ may have been due directly to changes in intracellular osmotic conditions (leading to greater or lesser water influx during hypo-osmotic stress), the magnitude of the changes indicates changes either in ion permeability or in membrane deformability. (As $V_{\text {hypo }}$ was measured $3 \mathrm{~min}$ after dilution in hypo-osmotic medium, the changes in $V_{\mathrm{r}}$ are unlikely to be due to changes in actual water permeability through the membrane since water permeability in spermatozoa is very rapid (see Gilmore et al., 1996 and references therein). Kulkarni et al. (1997) demonstrated that the bull sperm swelling response to hypoosmotic stress is dependent on the activity of a quininesensitive channel through which $\mathrm{K}^{+}$may exit so as to reduce and equilibrate intracellular osmolality. The activity of this channel may be, at least in part, under the control of the actin cytoskeleton (see Mills and Mandel, 1994). Noiles et al. (1997) found that treating mouse spermatozoa with cytochalasin D not only decreased F-actin concentrations within cells but also greatly affected the parameters of their volume response to hypo-osmotic stress. Therefore, the cyclical changes in $V_{\mathrm{r}}$ detected in the present study, like the changes in $V_{\text {iso' }}$ reflect changes in the dynamics of actin rearrangement and microfilament formation.

At present, as with the underlying basis for the volumetric changes themselves, an explanation for the enhanced cyclical behaviour of volume changes in spermatozoa in capacitating medium as compared with non-capacitating medium can only be guessed at. The cyclical changes may stem directly from the rapid changes in plasma membrane lipid architecture that occur in capacitating medium (Harrison et al., 1996). Alternatively, they may be due to the enhancement of cAMP concentrations in capacitating medium (Harrison, 1997) bringing about modifications in the actin cytoskeleton and, thence, on the quinine-sensitive $\mathrm{K}^{+}$channels (see Mills and Mandel, 1994).

The novel discovery of the potential for cyclical changes in sperm behaviour reported here has been based on analyses of the modal volume values in overall sperm populations. A problem when investigating behaviour of sperm populations is the heterogeneity of such populations and the difficulty of associating behavioural changes with particular subpopulations. The question arises as to whether the cyclical changes detected relate to a single subpopulation or are simply the net result of unilateral changes in successive cohorts of sperm undergoing capacitation (Bedford, 1970). Although even dead cells could not be distinguished within distributions obtained from iso-osmotic dilution, they could be largely distinguished and eliminated from analysis within the distributions resulting from hypo-osmotic dilution, because such damaged cells showed little or no swelling response and, therefore, contributed very little to the modal volume $V_{\text {hypo }}$ (this can be demonstrated by comparing the volume distributions of normal samples with samples that had been cold-shocked or permeabilized with digitonin; after hypo-osmotic dilution the untreated samples showed $V_{\text {hypo }}>>V_{\text {iso }}$ whereas the damaged samples showed $V_{\text {hypo }} \leqslant V_{\text {iso }}$ ). That the cyclical changes related to live cells was demonstrated by the observation that a diminishing subpopulation continued to express cyclical characteristics while increasing numbers of cells were becoming osmotically inactive. With respect to the possible contribution of succeeding cohorts, the number of cells within the analysed portion of the distribution remained essentially constant. Moreover, in samples from boars other than boar E, there was only occasional evidence of build-up of a subsidiary, osmotically inactive modal peak. Thus, while the counter-hypothesis that successions of subpopulations were responsible for cyclical characteristics within the sperm samples cannot be eliminated entirely, the present observations do not support this. Natural cyclical behaviour of cells is not unknown, and is considered to be brought about by subtle self-stabilizing feedback interactions between parallel control systems.

Petzoldt and Engel (1994) reported that differences in cell volume distribution among human sperm ejaculates could be related to conventional sperm parameters, and Petzoldt et al. (1994) suggested that volume measurements after hypoosmotic stress might be used to study the response of sperm membranes to preservation procedures. The present study has revealed clear differences between individual boars in terms of the amplitude and periodic parameters of the cyclical variation in the osmotic response of their spermatozoa. It was possible to divide the nine boars involved in the present study into two groups on this basis and these groups also differed significantly with respect to the decrease in motility during the in vitro incubation period. Although insufficient fertility data were available for any correlation to be made with the volumetric parameters, $V_{\mathrm{r}} \max$ was correlated with acrosomal loss during 
incubation, the tendency for $V_{\mathrm{r}}$ to decrease was correlated with the decrease in motility, and the duration of the first $V_{\mathrm{r}}$ cycle was correlated both with the decrease in motility and with acrosomal loss. Acrosomal loss and decrease in motility are both indicative of a tendency towards destabilization within the sperm population. Capacitation is considered a destabilizing process (see Harrison, 1996), and so it may be possible to use volumetric parameter values to indicate the destabilizing response of the sperm population to in vitro capacitating conditions. Therefore, the boars in group 2 would appear to have produced semen that undergoes capacitational destabilization more rapidly, or to a greater degree, than the semen from boars in group 1; indeed, the high $V_{\mathrm{r}} \max$ shown by the spermatozoa from group 2 boars may indicate a less stable disposition. However, at present, the implications of such a diagnosis are not clear. High response to in vitro capacitating conditions may be undesirable if the boar semen is to be used for conventional artificial insemination. In vivo, spermatozoa are usually inseminated $\geqslant 12 \mathrm{~h}$ before ovulation and must then survive in the female tract until the eggs arrive; a high tendency to destabilization in the environment of the female reproductive tract would lessen the likelihood of sufficient spermatozoa remaining fully active to fertilize the eggs once these are ovulated. Despite this uncertainty, parameters by which spermatozoa response to physiological conditions can be monitored in vitro are valuable potential tests of sperm fitness. Therefore, the discovery of the cyclical changes in the osmotic response of boar spermatoaoa indicates a hitherto undiscovered physiological property and a potential means by which boar reproductive quality might be judged.

This study was supported by the Karl-Eibl Fondation, Neustadt a.d. Aisch. The authors are most grateful to D. Waberski for much help in preparing the initial text of the manuscript.

\section{References}

Anderson TW (1971) The Statistical Analysis of Time Series J. Wiley, New York Bedford JM (1970) Sperm capacitation and fertilization in mammals Biology of Reproduction Supplement 2 128-158

Bredderman PJ and Foote RH (1971a) Alterations of cell volume in bull spermatozoa by factors known to affect active cation transport Experimental Cell Research 66 190-196

Bredderman PJ and Foote RH (1971b) Factors stabilizing bull sperm cell volume and prolonging motility at high dilution Experimental Cell Research $66458-464$

Castellani-Ceresa L, Brivio MF and Radaelli G (1992) F-actin in acrosomereacted boar spermatozoa Molecular Reproduction and Development 33 99-107

Drevius LO (1972) Bull spermatozoa as osmometers Journal of Reproduction and Fertility 28 29-39

Du J, Tao J, Kleinhaus FW, Peter AT and Critser JK (1994) Determination of boar spermatozoa water volume and osmotic response Theriogenology 42 1183-1191

Engel S and Petzoldt R (1994) The spermatozoal volume as indicative of the plasma membrane integrity (modification of the hypoosmotic swelling test) 1. Methods Andrologia 26 309-313

Florman HM, Arnoult C, Kazam IG, Li C and O'Toole CMB (1999) Calcium channels of mammalian sperm: properties and role in fertilization. In The Male Gamete. From Basic Science to Clinical Applications pp 187-193 Ed. C Gagnon. Cache River Press, Vienna, IL

Fox JEB and Boyles JK (1988) The membrane skeleton - a distinct structure that regulates the function of cells Bioessays 8 14-18
Gilmore JA, Du J, Tao J, Peter AT and Critser JK (1996) Osmotic properties of boar spermatozoa and their relevance to cryopreservation Journal of Reproduction and Fertility 107 87-95

Hancock JL (1956) The morphology of boar spermatozoa Journal of the Royal Microscopical Society 76 84-97

Harrison RAP (1988) Preliminary characterization of the multiple forms of ram sperm hyaluronidase Biochemical Journal 252 875-882

Harrison RAP (1996) Capacitation mechanisms, and the role of capacitation as seen in eutherian mammals Reproduction, Fertility and Development 8 581-594

Harrison RAP (1997) Sperm plasma membrane characteristics and boar semen fertility Journal of Reproduction and Fertility Supplement 52 195-211

Harrison RAP, Dott HM and Foster GC (1978) Effect of ionic strength, serum albumin and other macromolecules on the maintenance of motility and the surface of mammalian spermatozoa in a simple medium Journal of Reproduction and Fertility 52 65-73

Harrison RAP, Dott HM and Foster GC (1982) Bovine serum albumin, sperm motility, and the 'dilution effect' Journal of Experimental Zoology 222 81-88

Harrison RAP, Mairet B and Miller NGA (1993) Flow cytometric studies of bicarbonate-mediated $\mathrm{Ca}^{2+}$ influx in boar sperm populations Molecular Reproduction and Development 35 197-208

Harrison RAP, Ashworth PJC and Miller NGA (1996) Bicarbonate/ $\mathrm{CO}_{2}$, an effector of capacitation, induces a rapid and reversible change in the lipid architecture of boar sperm plasma membranes Molecular Reproduction and Development 45 378-391

Hoffmann EK and Dunham PB (1995) Membrane mechanisms and intracellular signalling in cell volume recognition International Reviews of Cytology 161 173-262

Krause D (1966) Untersuchungen am Bullensperma unter Berücksichtigung der fertilitätsdiagnostischen Bedeutung der Befunde Hannover, Tierärztliche Hochschule, Habilitationsschrift pp 52-56 School of Veterinary Medicine Hannover, Hannover

Kulkarni SB, Sauna ZE, Somlata V and Sitaramam V (1997) Volume regulation of spermatozoa by quinine-sensitive channels Molecular Reproduction and Development 46 535-550

Mills JW and Mandel LJ (1994) Cytoskeletal regulation of membrane transport events FASEB Journal 8 1161-1165

Moreno-Fierros L, Othon Hernandez E, Salgado ZO and Mujica A (1992) F-actin in guinea-pig spermatozoa: its role in calmodulin translocation during acrosome reaction Molecular Reproduction and Development 33 172-181

Müller H (1991) Wahrscheinlichkeitsrechnung und Mathematische Statistik Lexicon der Stochastik Akademie Verlag, Berlin

Noiles EE, Thompson KA and Storey BT (1997) Water permeability, L-p, of the mouse sperm plasma membrane and its activation energy are strongly dependent on interaction of the plasma membrane with the sperm cytoskeleton Cryobiology 35 79-92

Petzoldt R (1988) Elektronische Volumenanalyse - Eine Methode zur Beurteilung der Intaktheit der Spermienzellmembran Wissenschaftliche Zeitschrift Humboldt-Universität zu Berlin, Reihe Medizin 37 121-124

Petzoldt R and Engel S (1994) The spermatozoal volume as indicative of the plasma membrane integrity (modification of the hypoosmotic swelling test) 2. Diagnostic approach Andrologia 26 315-321

Petzoldt R, Waberski D, Kochel Ch and Weitze KF (1994) Zur Anwendbarkeit des computergestützen Schwelltestes bei Ebersperma für Routinelabor Reproduction in Domestic Animals 29203

Rink TJ (1977) Membrane potential of guinea-pig spermatozoa Journal of Reproduction and Fertility 51 155-157

SAS/STAT User's Guide (1989) Version 6, Fourth Edition, Vols 1 and 2. SAS Institute Inc, Cary, NC

Spungin B, Margalit I and Breitbart H (1995) Sperm exocytosis reconstructed in a cell-free system: evidence for the involvement of phospholipase $C$ and actin filaments in membrane fusion Journal of Cell Science 108 2525-2535

Töpfer-Petersen E, Enßlin M and Waberski D (1996) Kapazitation. Eine kurze Übersicht Fertilität 12 183-189

Yanagimachi R (1994) Mammalian fertilization. In The Physiology of Reproduction pp 189-317 Eds E Knobil and JD Neill. Raven Press, New York

Young IT (1977) Proof without prejudice: use of the Kolmogorov-Smirnov test for the analysis of histograms from flow systems and other sources Journal of Histochemistry and Cytochemistry 25 935-941

Zeng Y, Clark EN and Florman HM (1995) Sperm membrane potential: hyperpolarization during capacitation regulates zona pellucida-dependent acrosomal reaction Developmental Biology 171 554-563 


\section{Appendix}

\section{Normalization of the volumetric data}

The volume distributions were normalized to ensure that volumetric data were independent of the total cell number detected in the particular sampling. First, the original histogram $V(x)$ (where $x$ is the ongoing number of the effective particle size channel) was 'cut' at cursor positions $x_{1}$ and $x_{2}$, thus $V(x)=0$ for $x<x_{1}$, and $x>x_{2}$. Next, the area under the distribution curve was calculated between the intervals $x_{1}$ and $x_{2}$. Finally, the normalized histogram $V_{\text {norm }}(x)$ was built up from

$$
V_{\text {norm }}(x)=\frac{V(x)}{\int_{x_{1}}^{x_{2}} V(x) d x}
$$

Integration of equation A1 yields the value 1, that is, for every normalized volume distribution:

$$
\int_{x_{1}}^{x_{2}} V_{\text {norm }}(x)=1
$$

The integral of the volume distribution function $V(x)$ (that is, the divisor in (A1)) is a mathematical equivalent to the total number of cells involved in the actual measurement. For the normalized volume distribution function $V_{\text {norm }}(x)$, its integral (A2) is a constant (that is, 1) independent of the number of cells.

\section{Time series analysis}

Construction of model functions. The changes $V_{r}(t)$ and $V_{\text {iso }}(t)$ (that is, the time behaviours of the relative volume shift $V_{\mathrm{r}}$ and iso-osmotic volume $V_{\text {iso }}$ ) can be calculated on the basis of a periodic fluctuation model described by a finite Fourierseries (Anderson, 1971; Müller 1991):

$$
V(t)=\sum_{k=1}^{K} a_{k} \cdot \sin \left(\lambda_{k} t\right)+b_{k} \cdot \cos \left(\lambda_{k} t\right)+M(t)
$$

Given a time series of experimental values $V_{n}$ of the relative volume shift or of the iso-osmotic cell volume at the sampling points $t_{n}$ (for example, $t_{1}$ at $0 \mathrm{~min}, t_{2}$ at $15 \mathrm{~min}$ ), the frequencies $\lambda_{\mathrm{r}}$ valid for this model can be determined from the periodogram $I(\lambda)$ (Müller 1991):

$$
I(\lambda)=\frac{1}{2 \pi N}\left|\sum_{n=1}^{N} x_{n} e^{-i \lambda n}\right|^{2}
$$

The frequencies $\lambda_{\mathrm{\kappa}}$ granting $I\left(\lambda_{\mathrm{k}}\right) \geqslant I(\lambda)$ for all $\lambda_{\mathrm{\kappa}}$ can be calculated numerically from equation (A4) by varying $\lambda_{k}$ with respect to the scanning theorem (scanning rate $1 / 15$ $\left.\min ^{-1}\right), K$ being the number of solutions for $\lambda_{\mathrm{r}}$. Given the frequencies $\lambda_{\mathrm{k}^{\prime}}$ the coefficients $a_{\mathrm{k}^{\prime}} b_{\mathrm{k}}$ in equation (A3) can be calculated by regression analysis of experimental data $V_{n}$ and trigonometric functions $\sin \left(\lambda_{\mathrm{K}} t\right)$ and $\cos \left(\lambda_{\mathrm{K}} t\right) . M(t)$ is a non-periodic trend (usually polynomial or exponential). In the present study, the equations (A3) and (A4) were applied at the integer values $n=1,2, \ldots, N$ (where $N$ was the last sampling point).

Calculation of cycle durations. The maxima and minima of the model functions were used to determine the cycle durations of the volumetric oscillations. Time points $t_{m}$ yielding the local extreme values $V\left(t_{m}\right)$ (that is, the maximum or minimum value) of the model function $V(t)$ are given by solutions of the equation:

$$
\frac{d V_{\text {iso }}(t)}{d t}=0
$$

The solutions $t_{m}$ were calculated numerically by substituting the model function $V(t)$ according to equation (A3) into equation (A5) and varying $t$. Given all solutions of this equation over the time interval under consideration, the intervals between successive extreme values $t_{m}$ and $t_{m+1}$ (halfcycle durations) could be calculated as $T_{m}(1 / 2)=t_{m+1}-t_{m}$. With respect to the relative volume shift $V_{\mathrm{r}^{\prime}}$ the periods $T_{\mathrm{m}}$ for each individual boar were obtained by the sums of two successive half-cycle durations $T_{m-1}(1 / 2)+T_{m}(1 / 2)$. With respect to the iso-osmotic cell volume in suspensions during different incubation treatments, the mean period of $V(t)$ was determined by linear regression of $t_{m}$ and by number $m$ of the extreme value: from the regression model $t_{m}=\mathrm{a} m+\mathrm{b}$, it follows that $T_{m}(1 / 2)=t_{m+1}-t_{m}=a$ and $T_{m}=2 \mathrm{a}$.

\section{Calculation of volume equivalent}

The relationship between particle size channel $x$, effective diameter $D$ (in $\mu \mathrm{m}$ ) and estimated volume equivalent $V$ (in femtolitres) is given by equations (A6) and (A7):

$$
\begin{aligned}
& D=10 \cdot x / 1024 \\
& V=\frac{\pi}{6} \cdot D^{3} \cdot F
\end{aligned}
$$

where $F$ is a calibration factor obtained by measuring $V_{\text {iso }}$ for latex spheres of known size (Sigma; see Materials and Methods); $F=V_{\text {iso }}$ given $/ V_{\text {iso }}$ measured. 